\title{
SPORADIC AND IRRELEVANT PRIME DIVISORS
}

\author{
STEPHEN MCADAM AND L. J. RATLIFF, JR.
}

\begin{abstract}
Let $I$ represent a regular ideal in a Noetherian ring $R$. If $W$ is a finite set of prime ideals in $R$, some conditions on $W$ are given assuring that an $I$ can be found such that $W$ is exactly the set of primes which are in Ass $R / I$ but not in Ass $R / I^{n}$ for all large $n$. Furthermore, if $I$ is fixed, and if $P$ is a prime ideal containing $I$, some conditions are given assuring that in the Rees ring $\mathbf{R}=R[u, I t],(u, P, I t) \mathbf{R}$ is a prime divisor of $u \mathbf{R}$.
\end{abstract}

1. Introduction. This paper discusses two closely related topics. Let $I$ be a regular ideal in a Noetherian ring $R$. It is known that the sequence Ass $R / I$, Ass $R / I^{2}$,

Ass $R / I^{3}, \ldots$ eventually stabilizes to a set denoted $A^{*}(I)$. Thus Ass $R / I^{n}=A^{*}(I)$ for all large $n$. However, for small $n$ it may happen that there are prime ideals $P$ with $P \in$ Ass $R / I^{n}-A^{*}(I)$. Such a prime will be called a sporadic prime divisor of $I$. While various examples have appeared, there has never been a systematic study of such primes. Indeed, their nature leads us to suspect that there are limits to how much can be said concerning the sporadic prime divisors of an ideal. However, there is one not uncommon situation in which it is possible to find an ideal $K$ such that $A^{*}(K)=A^{*}(I)$, and Ass $R / K-A^{*}(K)=W$, where $W$ is a predetermined set, subject only to some mild restrictions. This will be presented in $\S 2$. $§ 3$ shows that if one is willing to sacrifice having $A^{*}(K)=A^{*}(I)$, one can get Ass $R / K-A^{*}(K)$ equaling a predetermined set $W$ in a very wide range of situations. In $\S 4$, we let $P$ be a prime containing $I$, and investigate situations in which in the Rees ring $\mathbf{R}=R[u, I t]$ of $R$ with respect to $I,(u, P, I t) \mathbf{R}$ is a prime divisor of $u \mathbf{R}$. We discuss the strong connections between the ideas in $\S 4$, and those in the earlier sections. $\S 5$ gives some examples of the results developed herein.

(1.1) Notation. $I$ will always be a regular ideal in a Noetherian ring $R$.

\section{Sporadic prime divisors.}

(2.1) Definitions. $A^{*}(I)=$ Ass $R / I^{n}$ for all large $n$. (See [B] or $[\mathbf{M}$, Chapter 1] to see that this is well defined.) For $n \geq 1$ let $S_{n}(I)=\operatorname{Ass} R / I^{n}-A^{*}(I)$. Let $S(I)=\bigcup S_{n}(I)$, over $n=1,2,3, \ldots$ We will call $S(I)$ the set of sporadic prime divisors of $I$. (Note that the existence of $A^{*}(I)$ shows that $S_{n}(I)=\varnothing$ for all sufficiently large $n$.) By $(I)_{a}$, we will mean the integral closure of $I$.

Received by the editors September 22, 1986.

1980 Mathematics Subject Classification (1985 Revision). Primary 13A15; Secondary 13B20.

Key words and phrases. Analytic spread, form ring, integral closure of an ideal, local ring, Noetherian ring, prime divisor, projectively equivalent ideals, reduction of an ideal, Rees ring, superficial element.

The second author was supported in part by the National Science Foundation, Grant DMS8521058. 
(2.2) LEMMA. Let $I^{*}$ denote the eventual stable value of the ascending chain $\left(I^{2}: I\right) \subseteq\left(I^{3}: I^{2}\right) \subseteq \cdots$.

(2.2.1) If $J$ is an ideal containing $I$, then $J \subseteq I^{*}$ if and only if there is an $n \geq 1$ with $J^{n}=I^{n}$.

(2.2.2) If $J$ is an ideal with $I \subseteq J \subseteq I^{*}$, then $A^{*}(J)=A^{*}(I)$.

(2.2.3) If $S$ is a multiplicatively closed subset of $R$ disjoint from $I$, then $\left(I_{S}\right)^{*}=$ $\left(I^{*}\right)_{S}$.

(2.2.4) $I \subseteq I^{*} \subseteq(I)_{a}$.

(2.2.5) For all large $n, I^{n^{*}}=I^{n}$.

(2.2.6) For $n \geq 1,\left(I^{*}\right)^{n} \subseteq I^{n^{*}}$.

(2.2.7) For $n \geq 1, I^{n^{*}}$ is the eventual stable value of the increasing chain $\left(I^{n+1}: I\right) \subseteq\left(I^{n+2}: I^{2}\right) \subseteq\left(I^{n+3}: I^{3}\right) \subseteq \cdots$.

(2.2.8) If $P \in \operatorname{Ass} R / I^{*}$, then $P \in \operatorname{Ass} R /\left(I^{*}\right)^{n}$ for all $n \geq 1$. Also, Ass $R / I^{*} \subseteq$ $A^{*}(I)$.

PROOF. (2.2.1), (2.2.4), and (2.2.5) are proved in [RR] (or in [M, Lemma 8.2]). (2.2.3) is straightforward. (2.2.2) is trivial from (2.2.1) and the definition of $A^{*}(I)$. (2.2.6) follows easily from (2.2.1). As for (2.2.7), the definition says that $I^{n^{*}}$ is the eventual stable value of $\left(I^{2 n}: I^{n}\right) \subseteq\left(I^{3 n}: I^{2 n}\right) \subseteq\left(I^{4 n}: I^{3 n}\right) \subseteq \cdots$. However, this is just a subchain of the chain in (2.2.7), and so both chains have the same stable value. Finally, for (2.2.8), suppose that $P \in \operatorname{Ass} R / I^{*}$. By (2.2.3), we may assume that $R$ is local at $P$. Write $P=\left(I^{*}: c\right)$ with $c \in R-I^{*}$. Obviously for $n \geq 1$, $P \subseteq\left(\left(I^{*}\right)^{n}: c\left(I^{*}\right)^{n-1}\right)$. We claim that equality holds. Since $P$ is a maximal ideal, we need only show that $\left(\left(I^{*}\right)^{n}: c\left(I^{*}\right)^{n-1}\right)$ is a proper ideal. If not, we would have $c \in\left(\left(I^{*}\right)^{n}:\left(I^{*}\right)^{n-1}\right) \subseteq\left(I^{*}\right)^{*}$ (by the definition). However, an easy exercise using (2.2.1) shows that $\left(I^{*}\right)^{*}=I^{*}$. Since $c \notin I^{*}$, we have a contradiction, proving the claim. The first part of (2.2.8) is immediate from the claim, while the second part is by the first part and (2.2.2).

While it may well happen that $I=I^{*}$, it is also true that for a large number of ideals, $I \neq I^{*}$, and it is these ideals in which we shall be interested. Given such an $I$, we will seek an ideal $K$ with $I \subseteq K \subseteq I^{*}$ such that $S_{1}(K)=W$, with $W$ a predetermined finite set of primes. Note that by $(2.2 .2), A^{*}(K)$ will equal $A^{*}(I)$. Thus, by moving from $I$ to $K$, we will leave the persistent prime divisors fixed, but will gain some control over the sporadic prime divisors. In our work, we will need three constraints on $W$. We now show the need for two of them.

(2.3) LEMmA. Let $K$ be an ideal with $I \subseteq K \subseteq I^{*}$, and let $P \in S_{1}(K)$. Then $P \notin A^{*}(I)$, and $\left(I: I^{*}\right) \subseteq P$.

Proof. Since $P \in S_{1}(K), P \notin A^{*}(K)$. Now (2.2.2) shows that $A^{*}(K)=A^{*}(I)$, and so $P \notin A^{*}(I)$, as desired. Next, suppose that $\left(I: I^{*}\right) \nsubseteq P$. Then it is easily seen that $I_{P}=\left(I^{*}\right)_{P}=I_{P}^{*}$ (by $(2.2 .3)$ ). As $I \subseteq K \subseteq I^{*}$, we must have $K_{P}=I_{P}^{*}$. Since $P \in S_{1}(K), P \in$ Ass $R / K$. Therefore, $P_{P} \in$ Ass $R_{P} / K_{P}=\operatorname{Ass} R_{P} / I_{P}^{*} \subseteq A^{*}\left(I_{P}\right)$ (using $(2.2 .8)$ ). This shows that $P \in A^{*}(I)$, which contradicts what we have just proved.

We can now state the main result of this section. Of the three conditions imposed on $W$ in (2.4), the need for two of them is explained by (2.3). The third condition (the most cumbersome of them), is needed to make an induction work. 
(2.4) THEOREM. Suppose that $I \neq I^{*}$. Let $W$ be a finite set of primes of $R$ with $W \cap A^{*}(I)=\varnothing$, and with $\left(I: I^{*}\right) \subseteq P$ for all $P \in W$. Furthermore, suppose that if $Q$ is a prime minimal over $\left(I: I^{*}\right)$ such that $\left(I: I^{*}\right)_{Q}=Q_{Q}$, then either $Q \notin W$ or $Q$ is maximal in $W$. (Note that if $Q \in W$, it must be minimal in $W$.) Then there is an ideal $K$ with $I \subseteq K \subseteq I^{*}$, and $S_{1}(K)=W$.

The proof of (2.4) requires two tools, one for forcing primes to be in Ass $R / J$, and one for excluding primes from Ass $R / J$, for certain ideals $J$. We now present these tools.

(2.5) LEMMA. Let $H$ be an ideal containing $I$, and let $V=\left\{P_{1}, \ldots, P_{n}\right\}$ be a finite set of prime ideals. Suppose that if $P$ is a minimal member of $V$, then $I_{P} \neq H_{P}$. Suppose also that if $P$ is a minimal member of $V$ which is not maximal in $V$, then $P_{P} H_{P} \nsubseteq I_{P}$. Let $J=I+\left(P_{1} \cdots P_{n}\right) H$. Then

(2.5.1) $V \subseteq$ Ass $R / J$.

(2.5.2) If $Q \in S_{1}(J)$, then either $P \subseteq Q$ for some $P \in V$ or $Q \in S_{1}(H)$.

ProOF. Let $P \in V$, and let $K$ be the product of those primes in $V$ which are properly contained in $P$. (If $P$ is minimal in $V, K=R$.) We claim that $I_{P}+K_{P} H_{P} \neq I_{P}$. If we have $P$ minimal in $V$, then $K=R$ and $I_{P}+K_{P} H_{P}=H_{P}$, so the claim follows from the hypothesis. If $P$ is not minimal in $V$, let $q \subset P$ with $q$ minimal in $V$. Note that $q$ is not maximal in $V$, and that $q$ is a factor of $K$. If our claim fails, then $I_{P}+K_{P} H_{P}=I_{P}$, and so localizing at $q_{P}$ gives $I_{q}+q_{q} H_{q}=I_{q}$. Thus $q_{q} H_{q} \subseteq I_{q}$. This contradicts our hypothesis, and proves the claim. Now Nakayama's Lemma shows that $I_{P}+P_{P}\left(I_{P}+K_{P} H_{P}\right) \subset I_{P}+K_{P} H_{P}$. Simplifying gives $I_{P}+P_{P} K_{P} H_{P} \subset I_{P}+K_{P} H_{P}$. Therefore, $\left(I_{P}+P_{P} K_{P} H_{P}: I_{P}+K_{P} H_{P}\right)$ is a proper ideal which obviously contains (and so equals) $P_{P}$. This shows that $P_{P}$ is a prime divisor of $I_{P}+P_{P} K_{P} H_{P}=J_{P}$. Thus $P \in \operatorname{Ass} R / J$, proving (2.5.1).

Next, suppose $Q \in S_{1}(J)$ and $P \nsubseteq Q$ for all $P \in V$. Then $J_{Q}=H_{Q}$. Since $Q \in$ $S_{1}(J)=$ Ass $R / J-A^{*}(J)$, we have $Q_{Q} \in \operatorname{Ass} R_{Q} / J_{Q}-A^{*}\left(J_{Q}\right)=\operatorname{Ass} R_{Q} / H_{Q}-$ $A^{*}\left(H_{Q}\right)$. Therefore, $Q \in S_{1}(H)$.

In the proof of (2.4), we will apply (2.5) to sets $V$ whose elements are pairwise incomparable, so that the assumption on primes $P$ which are minimal but not maximal in $V$ is not required.

(2.6) LEMMA. Let $H$ be an ideal, and let $I:\langle H\rangle$ denote the eventual stable value of $(I: H) \subseteq\left(I: H^{2}\right) \subseteq\left(I: H^{3}\right) \subseteq \cdots$. If $I=q_{1} \cap \cdots \cap q_{n}$ is a primary decomposition of $I$, and if $\operatorname{Rad} q_{i}$ contains $H$ exactly when $i \in\{1, \ldots, m\}$, then $I:\langle H\rangle=q_{m+1} \cap \cdots \cap q_{n}$ is a primary decomposition of $I:\langle H\rangle$. In particular, if $P \in$ Ass $R /(I:\langle H\rangle)$, then $H \nsubseteq P$.

PROOF. This is an easy exercise in primary decomposition.

PROOF OF (2.4). We first restate the hypotheses in the way we will use them. Clearly to say that $\left(I: I^{*}\right) \subseteq P$ is equivalent to saying $I_{P} \neq I_{P}^{*}$. Also, if $P$ is minimal but not maximal in $W$, the third hypothesis on $W$ is easily seen to say that $P_{P} I_{P}^{*} \nsubseteq I_{P}$.

For $P \in W$, define the $W$-height of $P$ to be the length of a longest chain of the form $P_{0} \subset P_{1} \subset \cdots \subset P_{n}=P$ with $P_{i} \in W$ for $i=0, \ldots, n$. Let $r$ be the maximum $W$-height of a prime in $W$, and for $i=0, \ldots, r$, let $W_{i}=\{P \in W \mid W$-height $P=i\}$ (so that $W_{0}$ is the set of minimal members of $W$ ). 
Let $I_{-1}=I^{*}$. We will inductively construct ideals $I_{0}, \ldots, I_{r}$, between $I$ and $I^{*}$, such that for $0 \leq i \leq r, S_{1}\left(I_{i}\right)=W_{0} \cup \cdots \cup W_{i}$. The result will follow from taking $K=I_{r}$. In our construction, we will have that $\left(I_{i}\right)_{q} \neq I_{q}$, for all $0 \leq i \leq r$ and all $q \in W_{0}$ with $q$ not maximal in $W$. (This will be needed to keep the induction going.)

Suppose we already have $I_{i-1}$ for $0 \leq i \leq r$ (recalling that $I_{-1}=I^{*}$ ). Before constructing $I_{i}$, we need an auxiliary ideal. We first claim that for $P \in W_{i}$ $\left(I_{i-1}\right)_{P} \neq I_{P}$. If instead $\left(I_{i-1}\right)_{P}=I_{P}$, then let $q \subseteq P$ with $q \in W_{0}$. Localizing at $q_{P}$ gives $\left(I_{i-1}\right)_{q}=I_{q}$. If $i=0$, then this says that $I_{q}^{*}=I_{q}$, which contradicts the hypothesis. If $i>0$, then $q \neq P$, and so $q$ is not maximal in $W$. Part of our inductive assumption on $I_{i-1}$ says that $\left(I_{i-1}\right)_{q} \neq I_{q}$, giving a contradiction. This proves the claim. Now let $J_{i}=I+w_{i} I_{i-1}$ with $w_{i}$ the product of the primes in $W_{i}$. The above claim, together with the fact that there are no containment relations among the primes in $W_{i}$, allows us to use (2.5.1) to see that $W_{i} \subseteq \operatorname{Ass} R / J_{i}$. We now claim that if $i>0$, then $W_{0} \cup \cdots \cup W_{i-1} \subseteq \operatorname{Ass} R / J_{i}$ as well. Let $Q \in W_{0} \cup \cdots \cup W_{i-1}$. Clearly $Q$ cannot contain any prime in $W_{i}$, and so $\left(w_{i}\right)_{Q}=R_{Q}$. Thus $\left(J_{i}\right)_{Q}=\left(I_{i-1}\right)_{Q}$. By induction, $W_{0} \cup \cdots \cup W_{i-1}=S_{1}\left(I_{i-1}\right) \subseteq$ Ass $R / I_{i-1}$. Therefore $Q_{Q} \in \operatorname{Ass} R_{Q} /\left(I_{i-1}\right)_{Q}=\operatorname{Ass} R_{Q} /\left(J_{i}\right)_{Q}$, so that $Q \in \operatorname{Ass} R / J_{i}$ as desired. We next claim that $W_{0} \cup \cdots \cup W_{i} \subseteq S_{1}\left(J_{i}\right)$. For this, we need only show that if $P \in W_{0} \cup \cdots \cup W_{i}$, then $P \notin A^{*}\left(J_{i}\right)$. However, since by induction $I \subseteq I_{i-1} \subseteq I^{*}$, clearly $I \subseteq J_{i} \subseteq I^{*}$. (2.2.2) shows that $A^{*}\left(J_{i}\right)=A^{*}(I)$, and the claim follows from the hypothesis that $W \cap A^{*}(I)=\varnothing$.

Let $U_{i}=S_{1}\left(J_{i}\right)-\left(W_{0} \cup \cdots \cup W_{i}\right)$, and let $P^{\prime} \in U_{i}$. We claim that $P^{\prime}$ properly contains a prime in $W_{i}$. If not, then since $P^{\prime} \notin W_{i}, P^{\prime}$ does not contain (properly or improperly) any prime in $W_{i}$. By (2.5.2), $P^{\prime} \in S_{1}\left(I_{i-1}\right)=W_{0} \cup \cdots \cup W_{i-1}$ (by induction). This contradicts that $P^{\prime} \in U_{i}$.

We now define $I_{i}=\left(J_{i}:\left\langle u_{i}\right\rangle\right) \cap I^{*}$, with $u_{i}$ the product of the primes in $U_{i}$. (If $U_{i}$ is empty, $u_{i}=R$.) Clearly $I \subseteq I_{i} \subseteq I^{*}$, as desired. Now let $q \in W_{0}$ with $q$ not maximal in $W$. We claim that $\left(I_{i}\right)_{q} \neq I_{q}$ (as is required in the third paragraph). Note that $q$ cannot contain any $P^{\prime} \in U_{i}$, since such a $P^{\prime}$ has been shown to properly contain a prime in $W_{i}$, and $q$ is minimal in $W$. Thus $\left(u_{i}\right)_{q}=R_{q}$, and $\left(I_{i}\right)_{q}=\left(J_{i}\right)_{q}$. However, $J_{i}=I+w_{i} I_{i-1}$. If $i=0$ (so that $I_{i-1}=I^{*}$ ) then $q$ is one of the factors of $w_{i}$, and so $\left(J_{i}\right)_{q}=I_{q}+q_{q} I_{q}^{*}$. By hypothesis (see the first paragraph), $q_{q} I_{q}^{*} \nsubseteq I_{q}$, and our claim is true in this case. On the other hand, let $i>0$. Then $\left(w_{i}\right)_{q}=R_{q}$, and $\left(J_{i}\right)_{q}=\left(I_{i-1}\right)_{q} \neq I_{q}$ by induction, proving the claim.

To complete the proof, it remains to show that $S_{1}\left(I_{i}\right)=W_{0} \cup \cdots \cup W_{i}$. Let $P \in W_{0} \cup \cdots \cup W_{i}$. Considering $W$-heights, we see that $P$ does not contain any prime $P^{\prime}$ in $U_{i}$, since we know that such a $P^{\prime}$ must properly contain a prime in $W_{i}$. Therefore, $\left(I_{i}\right)_{P}=\left(J_{i}\right)_{P}$. Since $P \in W_{0} \cup \cdots \cup W_{i} \subseteq S_{1}\left(J_{i}\right)$, we easily see that $P \in S_{1}\left(I_{i}\right)$. Thus, $W_{0} \cup \cdots \cup W_{i} \subseteq S_{1}\left(I_{i}\right)$. Conversely, let $Q \in S_{1}\left(I_{i}\right)$. Then $Q \in \operatorname{Ass} R / I_{i}$. Now a primary decomposition of $I_{i}$ can be obtained by intersecting a primary decomposition of $J_{i}:\left\langle u_{i}\right\rangle$ with a primary decomposition of $I^{*}$, and then deleting redundancies. Thus $Q$ must appear either in Ass $R /\left(J_{i}:\left\langle u_{i}\right\rangle\right)$ or in Ass $R / I^{*}$. Suppose $Q \in \operatorname{Ass} R / I^{*}$. Then (2.2.8) and (2.2.2) show that $Q \in$ Ass $R / I^{*} \subseteq A^{*}(I)=A^{*}\left(I_{i}\right)$, which contradicts that $Q \in S_{1}\left(I_{i}\right)$. Therefore, we must have $Q \in$ Ass $R /\left(J_{i}:\left\langle u_{i}\right\rangle\right)$. By (2.6), $u_{i} \nsubseteq Q$. Therefore, $\left(I_{i}\right)_{Q}=\left(J_{i}\right)_{Q}$. As 
$Q \in S_{1}\left(I_{i}\right)$, we get $Q \in S_{1}\left(J_{i}\right)=W_{0} \cup \cdots \cup W_{i} \cup U_{i}$. However, $Q$ cannot be in $U_{i}$, since we already have $u_{i} \nsubseteq Q$. Thus $Q \in W_{0} \cup \cdots \cup W_{i}$ as desired.

(2.7) COROLlary. Suppose $I \neq I^{*}$. Let $W$ be a finite set of primes with $W \cap A^{*}(I)=\varnothing$, and with $\left(I: I^{*}\right) \subseteq P$ for all $P \in W$. Furthermore, suppose that if $P$ is minimal but not maximal in $W$, then either of the following conditions holds.

(2.7.1) $P$ is not minimal over $\left(I: I^{*}\right)$.

(2.7.2) $P \notin$ Ass $R / I$.

Then there is an ideal $K$ with $I \subseteq K \subseteq I^{*}$ and $S_{1}(K)=W$.

PROOF. If $P$ is minimal over $\left(I: I^{*}\right)$, then $P \in \operatorname{Ass} R / I$. Thus (2.7.2) $\Rightarrow(2.7 .1)$, and we may assume that (2.7.1) holds for all $P$ minimal but not maximal in $W$. The result follows from (2.4).

Two cases warrant special mention. The first is when the primes in $W$ are pairwise incomparable. The second is when $\left(I: I^{*}\right)_{Q} \neq Q_{Q}$ for all primes $Q$ minimal over $\left(I: I^{*}\right)$. We treat these in the next two corollaries.

(2.8) COROllary. Suppose $I \neq I^{*}$. Let $W$ be a finite set of primes which are pairwise incomparable. Then there is an ideal $K$ with $I \subseteq K \subseteq I^{*}$ and $S_{1}(K)=W$ if and only if $W \cap A^{*}(I)=\varnothing$ and $\left(I: I^{*}\right) \subseteq P$ for all $P \in W$.

Proof. Suppose that $W \cap A^{*}(I)=\varnothing$ and $\left(I: I^{*}\right) \subseteq P$ for all $P \in W$. The $K$ we seek is given by (2.4), since every $Q \in W$ must be maximal in $W$. The converse is by (2.3).

(2.9) COROLlary. Suppose $I \neq I^{*}$. Suppose also that for any prime $Q$ minimal over $\left(I: I^{*}\right)$, we have $\left(I: I^{*}\right)_{Q} \neq Q_{Q}$. Let $W$ be any finite set of primes. Then there is an ideal $K$ with $I \subseteq K \subseteq I^{*}$ and $S_{1}(K)=W$ if and only if $W \cap A^{*}(I)=\varnothing$ and $\left(I: I^{*}\right) \subseteq P$ for all $P \in W$.

ProOF. One direction is by (2.4), and the other is by (2.3).

(2.10) REMARKS. (2.10.1) Even if $I=I^{*}$, it may happen that for some $n>1$, $I^{n} \neq I^{n^{*}}$. (However, by $(2.2 .5), n$ cannot be too large.) It does no harm to replace $I$ by $I^{n}$, since $A^{*}\left(I^{n}\right)=A^{*}(I)$. R. Cowsik has an (unpublished) example of a prime $P$ with $P$ the only prime divisor of $P^{n}$ for all $n \neq 2$, while $P^{2}$ has a prime divisor $Q \neq P$. Now (2.2.4) shows that $P=P^{*}$. If $P^{2}=P^{2^{*}}$, then by $(2.2 .8)$, $Q \in$ Ass $R / P^{2}=$ Ass $R / P^{2^{*}} \subseteq A^{*}\left(P^{2}\right)=A^{*}(P)=\{P\}$, a contradiction. Thus $P^{2} \neq P^{2^{*}}$.

(2.10.2) It may happen that $I^{n}=I^{n^{*}}$ for all $n \geq 1$. In that case, we can still make some progress by replacing $I$ by an ideal $H$ projectively equivalent to $I$ (although there is a small price to be paid). We discuss this in the next section.

3. Projectively equivalent ideals. In the preceding section, we dealt with an ideal $I$ for which $I \neq I^{*}$. As $(2.10 .1)$ points out, if $I^{n} \neq I^{n^{*}}$ for some $n \geq 1$, it does no harm to replace $I$ by $I^{n}$, since $A^{*}\left(I^{n}\right)=A^{*}(I)$. In this section, we will discuss replacing $I$ by an ideal $H$ projectively equivalent to $I$.

(3.1) DEFINITION. The ideals $I$ and $H$ are projectively equivalent if for some positive integers $n$ and $m,\left(I^{n}\right)_{a}=\left(H^{m}\right)_{a}$. Here $(J)_{a}$ is the integral closure of the ideal $J$.

There is a cost in replacing $I$ by $H$, since in general, $A^{*}(H) \neq A^{*}(I)$, so that in moving from $I$ to $H$, we have disturbed the persistent prime divisors. However, 
there is a limit to the cost. There are various important subsets of $A^{*}(I)$ which are not disturbed by this replacement. In particular, if $H$ is projectively equivalent to $I$, then $\bar{A}^{*}(H)=\bar{A}^{*}(I)$ and $E(H)=E(I)$. For facts regarding $\bar{A}^{*}(I)$ (the asymptotic prime divisors of $I$ ) see [M, Chapter 3]. Concerning $E(I)$ (the essential prime divisors of $I$ ), see $[\mathbf{K R}]$. (In $[\mathbf{K R}]$, the $E(I)$ is denoted $U(I)$ and called the $u$-essential primes.)

Because replacing $I$ by an ideal $H$ projectively equivalent to $I$ does not disturb $\bar{A}^{*}(I)$ or $E(I)$, such a replàcement is often useful. Therefore, in this section we shall investigate when we can find an $H$ projectively equivalent to $I$ such that $H \neq H^{*}$, so that the results of $\S 2$ can be applied to $H$. We will see that we very often can find such an $H$, even when $I^{n}=I^{n^{*}}$ for all $n \geq 1$.

Recall that if $J$ and $L$ are regular ideals of $R$, then $J$ is a reduction of $L$ (i.e., there is an $n \geq 1$ with $J L^{n}=L^{n+1}$ ) if and only if $J \subseteq L \subseteq(J)_{a}$ [NR1]. We will call $J$ a deep reduction of $L$ if $J$ is a reduction of $L$ and $J L \neq L^{2}$.

(3.2) Lemma. Let $J$ and $L$ be a regular ideals of $R$ with $J$ a deep reduction of $L$. Let $H=J L$. Then $H$ is projectively equivalent to $J$ and $H \neq H^{*}$.

Proof. Since $J \subseteq L \subseteq(J)_{a}$, clearly $J^{2} \subseteq J L \subseteq\left(J^{2}\right)_{a}$, so that $(J L)_{a}=\left(J^{2}\right)_{a}$. This shows that $H=J L$ is projectively equivalent to $J$. Now for some $n \geq 1$, $J L^{n}=L^{n+1}$. We see that $H^{n}=(J L)^{n}=\left(L^{2}\right)^{n}$. By $(2.2 .1), H \subseteq L^{2} \subseteq H^{*}$. Since $J$ is a deep reduction of $L, H=J L \neq L^{2}$. Thus, $H \neq H^{*}$, as desired.

Since for many ideals $I$, one can find a projectively equivalent ideal $J$ such that $J$ is a deep reduction of some ideal $L$ (quite often $L=(J)_{a}$ works), we can use (3.2) to find an $H$ projectively equivalent to $J$, and hence also to $I$, with $H \neq H^{*}$. The next result uses a variation of this idea.

(3.3) Lemma. Let $I=\left(a_{1}, \ldots, a_{n}\right)$ where $n \geq 2$. Let

$$
H=\left(a_{1}^{3}, \ldots, a_{n}^{3}\right)\left(a_{1}^{3}, \ldots, a_{n}^{3}, a_{1}^{2} a_{2}\right) .
$$

Then $H$ is projectively equivalent to $I$ and $H_{P} \neq H_{P}^{*}$ for any prime $P$ for which the images of $a_{1}, \ldots, a_{n}$ in $R_{P}$ are analytically independent. (Note that if height $I_{P}=n$, then $a_{1}, \ldots, a_{n}$ are analytically independent in $R_{P}$.)

Proof. Let $J=\left(a_{1}^{3}, \ldots, a_{n}^{3}\right)$, and let $L=\left(J, a_{1}^{2} a_{2}\right)$. Since $J \subseteq L \subseteq I^{3} \subseteq$ $\left(I^{3}\right)_{a}=(J)_{a}$, we see that $J$ is projectively equivalent to $I$ and also that $J$ is a reduction of $L$. It is easily seen that $H=J L$ is projectively equivalent to $J$, and so $H$ is projectively equivalent to $I$. Suppose that $a_{1}, \ldots, a_{n}$ are analytically independent in $R_{P}$. We need $H_{P} \neq H_{P}^{*}$. Since $H_{P}=J_{P} L_{P}$, and since $J_{P}$ is a reduction of $L_{P}$, by (3.2) it will suffice to show that $J_{P}$ is a deep reduction of $L_{P}$. However, $\left(a_{1}^{2} a_{2}\right)^{2}$ is in $\left(L_{P}\right)^{2}$, but is not in $J_{P} L_{P}$, using that $a_{1}, \ldots, a_{n}$ are analytically independent in $R_{P}$. Thus $J_{P} L_{P} \neq\left(L_{P}\right)^{2}$, as desired.

(3.4) COROLlaRY. Let $n \geq 2$, and let $I=\left(a_{1}, \ldots, a_{n}\right)$. Let

$$
H=\left(a_{1}^{3}, \ldots, a_{n}^{3}\right)\left(a_{1}^{3}, \ldots, a_{n}^{3}, a_{1}^{2} a_{2}\right) .
$$

Let $W$ be a finite set of primes such that $a_{1}, \ldots, a_{n}$ are analytically independent in $R_{P}$ for all $P \in W$. Suppose also that if $Q$ is a prime minimal over $\left(H: H^{*}\right)$ with $\left(H: H^{*}\right)_{Q}=Q_{Q}$, then either $Q \notin W$ or $Q$ is maximal in $W$. Finally suppose 
that $W \cap A^{*}(H)=\varnothing$. Then there is an ideal $K$ projectively equivalent to $I$ with $S_{1}(K)=W$.

Proof. By (3.3), we see that $H_{P} \neq H_{P}^{*}$ for all $P \in W$. Thus $\left(H: H^{*}\right) \subseteq P$ for all $P \in W$. Thus, all the hypotheses of $(2.4)$ are satisfied by $H$ and $W$, so there is a $K$ with $H \subseteq K \subseteq H^{*}$ and $S_{1}(K)=W$. Clearly $K$ is projectively equivalent to $I$, since $H$ is.

(3.5) PROPOSITION. Let $R$ be local and let the analytic spread of $I$ equal $n \geq 2$. Then there is an ideal $H$ projectively equivalent to $I$ with $H \neq H^{*}$.

PROOF. We claim that for some $m \geq 1, I^{m}$ has a reduction $B$ generated by $n$ elements. It is shown in [HO, Proposition 2] that $n$ is the least positive integer such that there are elements $c_{1}, \ldots, c_{n}$ in $R$ and positive integers $d_{1}, \ldots, d_{n}$, and $k$ such that $I^{k}=\sum c_{i} I^{k-d} i$, over $1 \leq i \leq n$. It then follows as in the proof of [NR2, Theorems 3, 4] that if $m=d_{1} \cdots d_{n}, e_{i}=m / d_{i}$, and $a_{i}=\left(c_{i}\right)^{e} i$, then $B=\left(a_{1}, \ldots, a_{n}\right) R$ is a reduction of $I^{m}$, proving the claim.

Let $R\langle X\rangle=R[X]_{M R \mid X]}$. Then $B R\langle X\rangle$ is a reduction of $I^{m} R\langle X\rangle$. Since the analytic spread of $I^{m} R\langle X\rangle$ is still $n$, and since $B R\langle X\rangle$ is generated by $n$ elements, the fact that the local ring $R\langle X\rangle$ has infinite residue field allows us to conclude that $B R\langle X\rangle$ is a minimal reduction of $I^{m} R\langle X\rangle$, and that $a_{1}, \ldots, a_{n}$ are analytically independent in $R\langle X\rangle$. It follows that $a_{1}, \ldots, a_{n}$ are analytically independent in $R$ (and that $B$ is a minimal reduction of $I^{m}$ ). Let $H$ be as in (3.3). Then $H \neq H^{*}$, and $H$ is projectively equivalent to $B$, and hence to $I$, and we are done.

(3.6) REMARK. Since for $j \geq 1,\left(a_{1}^{j}, \ldots, a_{n}^{j}\right) R$ is a reduction of $I^{m j}$, the above argument shows $\left(a_{1}^{j}, \ldots, a_{n}^{j}\right) R$ is a minimal reduction of $I^{m j}$. Thus infinitely many powers of $I$ have minimal reductions (even when $n=1$ ).

(3.7) REMARK. Let $I=b R$ with $b$ regular and $R$ either normal, or as in $[\mathbf{N}$, Example 2, pp. 203-205]. Then $H=H^{*}$ for any $H$ projectively equivalent to $I$. We know of no other cases of this behavior.

\section{Irrelevant prime divisors.}

(4.1) Definitions. (4.1.1) The Rees ring of $R$ with respect to $I$ is $\mathbf{R}(I)=$ $R[u, I t]$ where $t$ is an indeterminate and $u=t^{-1} . \mathbf{F}(R, I)=R / I+I / I^{2}+I^{2} / I^{3}+\cdots$ is the form ring of $R$ with respect to $I$. A prime $Q$ in a graded ring is irrelevant if it contains all homogeneous elements of positive degree. Otherwise, $Q$ is relevant.

(4.1.2) An element $b$ is a superficial element (of degree $k \geq 1$ ) for $I$ if $b \in I^{k}$ and there is an $m \geq 1$ such that $\left(I^{n+k}: b\right) \cap I^{m}=I^{n}$ for all large $n$. If in fact $I^{n+k}: b=I^{n}$ for all $n \geq 1$, we say that $b$ is strongly superficial.

(4.2) REMARK. It is easily seen that $\mathbf{F}(R, I)$ is isomorphic to $\mathbf{R}(I) / u \mathbf{R}(I)$. If $Q \in \operatorname{Spec} \mathbf{R}$, then $Q$ is an irrelevant prime divisor of $u \mathbf{R}$ if and only if $Q / u \mathbf{R}$ is an irrelevant prime divisor of zero in $\mathbf{F}(R, I)$. The following results can be stated in terms of either the Rees ring or the form ring. We opt for the former.

(4.3) and (4.5) show the relevance of irrelevant primes to the material discussed in the previous sections.

(4.3) ThEOREM. Let $I \subseteq P \in \operatorname{Spec} R$, and let $\mathbf{R}=\mathbf{R}(I)$. Then

(4.3.1) $P \in \operatorname{Ass} R / I^{n}$ for some $n \geq 1$ if and only if there is a $Q \in \operatorname{Ass} \mathbf{R} / u \mathbf{R}$ with $Q \cap R=P$. 
(4.3.2) $P \in A^{*}(I)$ if and only if there is a relevant prime $Q \in$ Ass $\mathbf{R} / u \mathbf{R}$ with $Q \cap R=P$.

(4.3.3) $P \in S(I)$ if and only if $(u, P, I t)$ is the only prime divisor of $u \mathbf{R}$ which lies over $P$.

Proof. (4.3.1) Let $P \in \operatorname{Ass} R / I^{n}$. Since $u^{n} \mathbf{R} \cap R=I^{n}, P$ lifts to a prime divisor $Q$ of $u^{n} \mathbf{R}$. Since $u$ is regular in $\mathbf{R}, Q \in$ Ass $\mathbf{R} / u \mathbf{R}$. Conversely, suppose that $Q \in$ Ass $\mathbf{R} / u \mathbf{R}$. Since $u \mathbf{R}$ is homogeneous, there is a homogeneous element $c t^{k}$ with $Q=\left(u \mathbf{R}: c t^{k}\right)$. Certainly $k \geq 0$, since $c t^{k} \notin u \mathbf{R}$. Therefore, $P=Q \cap R=$ $\left(u \mathbf{R}: c t^{k}\right) \cap R=\left(I^{k+1}: c\right)$, showing $P \in \operatorname{Ass} R / I^{k+1}$.

Since $I$ is regular, (4.3.2) is proved in [M, Propositions 1.15, 2.2].

(4.3.3) follows easily from (4.3.1) and (4.3.2) and the fact that $(u, P, I t)$ is the largest homogeneous prime in $\mathbf{R}$ which lies over $P$.

(4.4) LemmA. Let $\mathbf{R}=\mathbf{R}(I)$, and let $m \geq 1$ and $k \geq 1$ be integers. When $j \leq 0$, let $I^{j}=R$ and $I^{j^{*}}=R$. Then

(4.4.1) $u^{m} \mathbf{R}:(I t \mathbf{R})^{k}$ has the form $\sum\left(\left(I^{m+k+n}: I^{k}\right) \cap I^{n}\right) t^{n}$, over all integers $n$.

(4.4.2) $u^{m} \mathbf{R}:\langle I t \mathbf{R}\rangle$ has the form $\sum\left(\left(I^{m+n}\right)^{*} \cap I^{n}\right) t^{n}$, over all integers $n$.

PROOF. (4.4.1) is straightforward. For (4.4.2), let $J(k, n)=\left(I^{m+k+n}: I^{k}\right) \cap$ $I^{n}$, so that $u^{m} \mathbf{R}:(I t \mathbf{R})^{k}=\sum J(k, n) t^{n}$. Now the sequence $\left(u^{m} \mathbf{R}: I t \mathbf{R}\right) \subseteq$ $\left(u^{m} \mathbf{R}:(I t \mathbf{R})^{2}\right) \subseteq\left(u^{m} \mathbf{R}:(I t \mathbf{R})^{3}\right) \subseteq \cdots$ eventually stabilizes to $u^{m} \mathbf{R}:\langle I t \mathbf{R}\rangle$. Let $k$ be large enough that this stabilization has occurred. Thus $u^{m} \mathbf{R}:\langle$ It $\mathbf{R}\rangle=$ $\sum J(k, n) t^{n}$. It follows that for each integer $n$, the sequence $J(1, n) \subseteq J(2, n) \subseteq$ $J(3, n) \subseteq \cdots$ stabilizes to $J(k, n)$. However, (2.2.7) shows that this last sequence stabilizes to $\left(I^{m+n}\right)^{*} \cap I^{n}$. The result follows.

The reader might be interested in $[\mathbf{M R},(2.4)]$ in which $R\left[u, I^{*} t, I^{2^{*}} t^{2}, I^{3^{*}} t^{3}, \ldots\right]$ appears.

(4.5) Proposition. Let $\mathbf{R}=\mathbf{R}(I)$. The following are equivalent.

(4.5.1) $u \mathbf{R}$ has no irrelevant prime divisors.

(4.5.2) $I^{n}=I^{n^{*}}$ for all $n \geq 1$.

(4.5.3) I has a strongly superficial element.

(4.5.4) $\left(I^{n+2}: I\right) \cap I^{n}=I^{n+1}$ for all $n \geq 0$.

(4.5.5) There is a $k \geq 1$ with $\left(I^{n+k}: I^{k}\right)=I^{n}$ for all $n \geq 1$.

PROOF. (4.5.1) $\Rightarrow(4.5 .3)$ If $u \mathbf{R}$ has no irrelevant prime divisors, then a fairly wellknown graded version of the prime avoidance lemma allows us to find an element $b t^{k}$ of $I t \mathbf{R}$ with $b t^{k}$ a nonzero divisor modulo $u \mathbf{R}$. Of course $k \geq 1$, and $b \in I^{k}$. For all $n \geq 1, u^{n} \mathbf{R}: b t^{k}=u^{n} \mathbf{R}$. Intersecting with $R$ shows that $I^{n+k}: b=I^{n}$. Thus $b$ is strongly superficial of degree $k$ for $I$.

(4.5.3) $\Rightarrow(4.5 .5)$ Let $b$ be a strongly superficial element of degree $k$ for $I$. For any $n \geq 1, I^{n} \subseteq\left(I^{n+k}: I^{k}\right) \subseteq\left(I^{n+k}: b\right)=I^{n}$, and so (4.5.5) holds.

(4.5.5) $\Rightarrow(4.5 .2)$ Let $(4.5 .5)$ hold, and let $n \geq 1$. We claim that for any $m \geq 1$, $\left(I^{n+m k}: I^{m k}\right)=I^{n}$. To see this, we induct on $m$, the key equation being

$$
\left(I^{n+m k}: I^{m k}\right)=\left(\left(I^{n+m k}: I^{k}\right): I^{(m-1) k}\right)=\left(I^{n+(m-1) k}: I^{(m-1) k}\right),
$$

the last equality by (4.5.5). As (2.2.7) shows that for large $m$ we have $\left(I^{n+m k}\right.$ : $\left.I^{m k}\right)=I^{n^{*}}$, we see that $(4.5 .2)$ holds. 
(4.5.2) $\Rightarrow(4.5 .4)$ Since $I^{n+1} \subseteq\left(I^{n+2}: I\right) \subseteq I^{n+1^{*}}$, if (4.5.2) holds, then (4.5.4) easily follows.

(4.5.4) $\Rightarrow(4.5 .1)$ By (4.4.1) (with $r_{1}=k=1$ ), we have

$$
u \mathbf{R}: I t \mathbf{R}=\sum\left(\left(I^{n+2}: I\right) \cap I^{n}\right) t^{n}
$$

over all integers $n$. If (4.5.4) holds, then this last expression equals $\sum I^{n+1} t^{n}=u \mathbf{R}$. Thus, $u \mathbf{R}: I t \mathbf{R}=u \mathbf{R}$ showing that $I t \mathbf{R}$ does not consist of zero divisors modulo $u \mathbf{R}$. It follows that (4.5.1) holds.

(4.6) REMARKS. (4.6.1) A direct proof of $(4.5 .1) \Leftrightarrow(4.5 .2)$ is easily fashioned from (4.4.2).

(4.6.2) The arguments for $(4.5 .2) \Rightarrow(4.5 .4) \Rightarrow(4.5 .1)$ would work as well if (4.5.4) said that there are positive integers $m$ and $k$ with $\left(I^{m+k+n}: I^{k}\right) \cap I^{n}=I^{m+n}$ for all $n \geq 1-m$. Thus this statement is equivalent to the others. (Note this statement differs from that of (4.5.5) only by the intersection with $I^{n}$.)

(4.6.3) The equivalence $(4.5 .1) \Leftrightarrow(4.5 .3)$ first appeared in $[\mathbf{S}]$.

In the following circumstance, we can strengthen (4.5.3).

(4.7) Proposition. Let $(R, M)$ be local with $R / M$ infinite. Then the statements (4.5.1) through (4.5.5) hold if and only if $I$ has a minimal reduction $\left(b_{1}, \ldots, b_{m}\right) R$ with each $b_{i}$ a strongly superficial element of degree 1 for $I$.

PROOF. If (4.5.1) holds, then the proof of [NR1, Lemma 1, p. 152] shows that $I$ has a minimal reduction $\left(b_{1}, \ldots, b_{m}\right) R$ with $b_{i} t$ a nonzero divisor modulo $u \mathbf{R}, i=1, \ldots, m$. Consider some $b_{i}$, and some $n \geq 1$. Let $c \in\left(I^{n+1}: b_{i}\right)$. Then $c \in\left(u^{n} \mathbf{R}: b_{i} t\right) \cap R$. However, $b_{i} t$ is a nonzero divisor modulo $u^{n} \mathbf{R}$, and so $c \in u^{n} \mathbf{R} \cap R=I^{n}$. Thus $I^{n+1}: b_{i}=I^{n}$, so that $b_{i}$ is a strongly superficial element of degree 1 for $I$. The converse is by (4.5).

Let $P$ be a prime containing $I$. Then $(u, P, I t) \mathbf{R}$ is an irrelevant prime of $\mathbf{R}=$ $\mathbf{R}(I)$. We consider the interesting question of determining when $(u, P, I t) \mathbf{R}$ is a prime divisor of $u \mathbf{R}$. Of course if $P$ is a sporadic prime divisor of $I$, then (4.3.3) tells us that $(u, P, I t) \mathbf{R}$ must be a prime divisor of $u \mathbf{R}$. Furthermore, (4.5) give us some circumstances in which $(u, P, I t) \mathbf{R}$ cannot be a prime divisor of $u \mathbf{R}$. We now consider some other results relating to this question. In what follows, $\mathbf{R}$ will always denote $\mathbf{R}(I)$, and $P \in \operatorname{Spec} R$ with $I \subseteq P$.

(4.8) REMARK. Let $S=R-P$. Then $\mathbf{R}_{S}=R_{S}\left[u, I_{S} t\right]$ is the Rees ring of $R_{S}$ with respect to $I_{S}$. Also, $((u, P, I t) \mathbf{R})_{S}=\left(u, P_{S}, I_{S} t\right) \mathbf{R}_{S}$. Thus in studying when $(u, P, I t) \mathbf{R}$ is a prime divisor of $u \mathbf{R}$, we may assume that $R$ is local at $P$.

(4.9) REMARK. The sequence Ass $R / I$, Ass $I / I^{2}$, Ass $I^{2} / I^{3}, \ldots$ eventually stabilizes, and since $I$ is regular, its eventual stable value is $A^{*}(I)$, as is shown in [B] or [M, Chapter 1].

(4.10) LEMMA. Suppose that for some $n \geq 1, P=\left(I^{n}: b\right)$ (so that $\left(u^{n} \mathbf{R}\right.$ : $b \mathbf{R}) \subseteq(u, P, I t) \mathbf{R})$. Suppose also that $(u, P, I t) \mathbf{R}$ is not minimal over $\left(u^{n} \mathbf{R}: b \mathbf{R}\right)$. Then for all $k \geq 0, P \in$ Ass $R / I^{n+k}$. If furthermore $b \in I^{n-1}$, then for all $k \geq 0$, $P \in \operatorname{Ass} I^{n+k-1} / I^{n+k}$. (Note that the assumption on $(u, P, I t) \mathbf{R}$ is fulfilled if that prime is not a prime divisor of $u \mathbf{R}$.)

PROOF. Since $\left(u^{n} \mathbf{R}: b \mathbf{R}\right) \cap R=\left(I^{n}: b\right)=P$, there is a prime $Q$ of $\mathbf{R}$ minimal over $\left(u^{n} \mathbf{R}: b \mathbf{R}\right)$ with $Q \cap R=P$. Since $(u, P, I t) \mathbf{R}$ contains any homogeneous 
ideal which intersects $R$ at $P$, and since $Q$ is a prime divisor of $u^{n} \mathbf{R}$, and hence is homogeneous, we have $Q \subseteq(u, P, I t) \mathbf{R}$. By hypothesis, $Q$ cannot equal $(u, P, I t) \mathbf{R}$, and therefore we must have $I t \nsubseteq Q$. Let $c \in I$, with $c t \notin Q$. For any $k \geq 0$,

$$
\left(u^{n} \mathbf{R}: b(c t)^{k} \mathbf{R}\right)=\left(\left(u^{n} \mathbf{R}: b \mathbf{R}\right):(c t)^{k} \mathbf{R}\right) \subseteq\left(Q:(c t)^{k} \mathbf{R}\right)=Q .
$$

Contracting to $R$ shows that $\left(I^{n+k}: b c^{k}\right) \subseteq P$. However, $P \subseteq\left(I^{n+k}: b c^{k}\right)$ since $P=\left(I^{n}: b\right)$ and $c \in I$. Thus $P=\left(I^{n+k}: b c^{k}\right)$, so that $P \in \operatorname{Ass} R / I^{n+k}$. If $b \in I^{n-1}$, then $b c^{k} \in I^{n+k-1}$, and so $P \in \operatorname{Ass} I^{n+k-1} / I^{n+k}$.

(4.11) Proposition. (4.11.1) If for some $n \geq 1, P \in A s s R / I^{n}$ but $P \notin$ Ass $R / I^{n+1}$ (respectively, $P \in \operatorname{Ass} I^{n-1} / I^{n}$ but $\left.P \notin \operatorname{Ass} I^{n} / I^{n+1}\right)$ then $(u, P, I t) \mathbf{R}$ is a prime divisor of $u \mathbf{R}$.

(4.11.2) If for some $n \geq 1, P \in \operatorname{Ass} R / I^{n}$ but $P \notin \operatorname{Ass} R / I^{n^{*}}$, then $(u, P, I t) \mathbf{R}$ is a prime divisor of $u \mathbf{R}$.

PROOF. (4.11.1) follows immediately from (4.10) (using the parenthetical statement). As for (4.11.2), suppose that $P \in \operatorname{Ass} R / I^{n}$ but $P \notin \operatorname{Ass} R / I^{n^{*}}$. Let $q_{1} \cap \cdots \cap q_{r} \cap q_{r+1} \cap \cdots \cap q_{s}$ be a primary decomposition of $u^{n} \mathbf{R}$ with $q_{i}$ primary to $Q_{i}$, and suppose the ordering is such that $Q_{i}$ is relevant for $i=1, \ldots, r$. By (4.3.1) (and the fact that $u$ is regular in $\mathbf{R}$ ), we see that some of our primes $Q_{i}$ must lie over $P$. To prove our result, we must show that for some $i=r+1, \ldots, s, Q_{i}$ lies over $P$, so that $Q_{i}=(u, P, I t) \mathbf{R}$. If not, then we may assume that $Q_{1}, \ldots, Q_{w}$ are the ones lying over $P$, and that $w \leq r$ (and we will get a contradiction). Since $q_{i} \cap R$ is primary to $Q_{i} \cap R$, and since $u^{n} \mathbf{R} \cap R=I^{n}$, we see that $\left(q_{1} \cap R\right) \cap \cdots \cap\left(q_{r} \cap R\right) \cap\left(q_{r+1} \cap\right.$ $R) \cap \cdots \cap\left(q_{s} \cap R\right)=I^{n}$. Let $J=\left(q_{1} \cap R\right) \cap \cdots \cap\left(q_{w} \cap R\right)$, so that $J$ is primary to $P$. Then $J \cap\left(q_{w+1} \cap R\right) \cap \cdots \cap\left(q_{r} \cap R\right) \cap\left(q_{r+1} \cap R\right) \cap \cdots \cap\left(q_{s} \cap R\right)=I^{n}$, and by deleting redundancies, this will lead to a primary decomposition of $I^{n}$. Since $P \in \operatorname{Ass} R / I^{n}$, the $J$ cannot be deleted. Therefore, $\left(q_{w+1} \cap R\right) \cap \cdots \cap\left(q_{r} \cap R\right) \cap\left(q_{r+1} \cap R\right) \cap \cdots \cap\left(q_{s} \cap R\right)$ is not contained in $J$.

On the othel hand, by (2.6), $q_{1} \cap \cdots \cap q_{r}$ is a primary decomposition of $u^{n} \mathbf{R}$ : $\langle I t \mathbf{R}\rangle$. Since (4.4.2) shows that $\left(u^{n} \mathbf{R}:\langle I t \mathbf{R}\rangle\right) \cap R=I^{n^{*}}$, we see that $\left(q_{1} \cap R\right) \cap \cdots \cap$ $\left(q_{r} \cap R\right)=I^{n^{*}}$. Thus $J \cap\left(q_{w+1} \cap R\right) \cap \cdots \cap\left(q_{r} \cap R\right)=I^{n^{*}}$, and deleting redundancies leads to a primary decomposition of $I^{n^{*}}$. However, since $P \notin$ Ass $R / I^{n^{*}}$, we see that the $J$ must be deleted. Thus, $\left(q_{w+1} \cap R\right) \cap \cdots \cap\left(q_{r} \cap R\right) \subseteq J$. This contradicts the final sentence of the previous paragraph.

Suppose that $I^{n} \neq I^{n^{*}}$ for some $n \geq 1$. Then (4.5.1) $\Rightarrow(4.5 .2)$ shows that $u \mathbf{R}$ has at least one irrelevant prime divisor, but does not identify any of them. The next result shows how, in this case, to identify at least one irrelevant prime divisor of $u \mathbf{R}$.

(4.12) Proposition. Assume $I^{n} \neq I^{n^{*}}$ for some $n \geq 1$, and let $P$ be a prime divisor of $\left(I^{n}: I^{n^{*}}\right)$. Then $(u, P, I t) \mathbf{R}$ is a prime divisor of $u \mathbf{R}$.

PROOF. Suppose this is false. Let $q_{1}, \ldots, q_{w}, q_{w+1}, \ldots, q_{r}, q_{r+1}, \ldots, q_{s}$, and $J$ be as in the proof of (4.11.2). Note that since $P$ is a prime divisor of $\left(I^{n}: I^{n^{*}}\right), P$ is a prime divisor of $I^{n}$, and so (4.3.1) shows $w \geq 1$ (and $w \leq r$ by supposition). If we let $L=\left(q_{w+1} \cap R\right) \cap \cdots \cap\left(q_{r} \cap R\right) \cap\left(q_{r+1} \cap R\right) \cap \cdots \cap\left(q_{s} \cap R\right)$, then the previous proof shows that $I^{n}=J \cap L$. However, the second paragraph of that proof shows that $I^{n^{*}} \subseteq J$. Therefore, $\left(I^{n}: I^{n^{*}}\right)=\left(J \cap L: I^{n^{*}}\right)=\left(L: I^{n^{*}}\right)$. Now $P$ is a prime 
divisor of $\left(I^{n}: I^{n^{*}}\right)=\left(L: I^{n^{*}}\right)$, and so $P$ is a prime divisor of $L$. However, clearly the prime divisors of $L$ come from among the set $Q_{i} \cap R$, for $w+1 \leq i \leq s$, and by the ordering, $P$ is not one of these. This is a contradiction.

(4.13) Questions. (4.13.1) Does the converse of (4.12) hold? That is, if $(u, P, I t) \mathbf{R}$ is a prime divisor of $u \mathbf{R}$, must $P$ be a prime divisor of $\left(I^{n}: I^{n^{*}}\right)$ for some $n \geq 1$ ?

(4.13.2) If for some $n \geq 1, P \in \operatorname{Ass} R / I^{n}$ but $P \notin$ Ass $R / I^{n^{*}}$, does it follow that $P$ is a prime divisor of $\left(I^{n}: I^{n^{*}}\right)$ ? (Example (5.2) shows that the converse to this question is false, since in that example, $P$ is a prime divisor of each of $\left(I: I^{*}\right), I$, and $I^{*}$.)

(4.14) Proposition. (4.14.1) [R, (3.3)] There is a prime divisor of $u \mathbf{R}$ containing $(u, P, I t) \mathbf{R}$ if and only if for some $n \geq 0, I^{n+1}$ is properly contained in $I^{n} \cap\left(I^{n+2}: I\right) \cap\left(I^{n+1}: P\right)$.

(4.14.2) $(u, P, I t) \mathbf{R}$ is a prime divisor of $u \mathbf{R}$ if and only if for some $n \geq 0$, $P=\left(I^{n+1}: b\right)$ for some $b \in\left(I^{n+2}: I\right) \cap I^{n}$.

ProOF. (4.14.1) $(u, P, I t) \mathbf{R}$ is contained in some prime divisor of $u \mathbf{R}$ if and only if there is an element $b t^{n} \in \mathbf{R}-u \mathbf{R}$ with $(u, P, I t) \mathbf{R}\left(b t^{n}\right) \subseteq u \mathbf{R}$. Now $b t^{n} \in \mathbf{R}-u \mathbf{R}$ if and only if $b \in I^{n}-I^{n+1}$ (which requires $n \geq 0$ ). Also, $I t\left(b t^{n}\right) \subseteq u \mathbf{R}$ if and only if $b \in\left(I^{n+2}: I\right)$, and $P\left(b t^{n}\right) \subseteq u \mathbf{R}$ if and only if $b \in\left(I^{n+1}: P\right)$. Together, these show that $(u, P, I t) \mathbf{R}$ is contained in some prime divisor of $u \mathbf{R}$ if and only if there is an element $b$ of $R$ with $b \in\left(I^{n} \cap\left(I^{n+2}: I\right) \cap\left(I^{n+1}: P\right)\right)-I^{n+1}$, giving (4.14.1).

(4.14.2) As both the hypothesis and conclusion of (4.14.2) localize well, we may assume that $R$ is local at $P$. Now $(u, P, I t) \mathbf{R}$ is the unique maximal homogeneous ideal of $\mathbf{R}$, so (4.14.1) shows that $(u, P, I t) \mathbf{R}$ is a prime divisor of $u \mathbf{R}$ if and only if there is an $n \geq 0$ and a $b \in I^{n} \cap\left(I^{n+2}: I\right) \cap\left(I^{n+1}: P\right)$ with $b \notin I^{n+1}$. If such exist, then $b \in\left(I^{n+1}: P\right)$ shows that $P \subseteq\left(I^{n+1}: b\right)$. However, $b \notin I^{n+1}$ and $(R, P)$ local shows that $\left(I^{n+1}: b\right) \subseteq P$. Thus $P=\left(I^{n+1}: b\right)$ with $b \in I^{n} \cap\left(I^{n+2}: I\right)$. This shows one direction of (4.14.2). For the converse, suppose $P=\left(I^{n+1}: b\right)$ with $b \in I^{n} \cap\left(I^{n+2}: I\right)$. Since $\left(I^{n+1}: b\right)=P$, we have $b \notin I^{n+1}$. However, $b \in I^{n} \cap\left(I^{n+2}: I\right) \cap\left(I^{n+1}: P\right)$, and we are done.

Our final result in this section discusses the sequence $I^{*}, I^{2^{*}}, I^{3^{*}}, \ldots$ We still assume that $I \subseteq P \in \operatorname{Spec} R$.

(4.15) Proposition. (4.15.1) Suppose that $0 \leq m<n$. Then

$$
\left(I^{n^{*}}: I^{m^{*}}\right)=\left(I^{n^{*}}: I^{m}\right)=I^{n-m^{*}} .
$$

(4.15.2) For $n \geq 1$, let $J$ be an ideal with $J \subseteq I^{n^{*}}$. If $P \in$ Ass $R / J$, then $P \in \operatorname{Ass} I^{n-1^{*}} / J$.

(4.15.3) Ass $R / I^{*} \subseteq$ Ass $R / I^{2^{*}} \subseteq$ Ass $R / I^{3^{*}} \subseteq \cdots$.

(4.15.4) For $n \geq 1, S_{n}(I) \subseteq \operatorname{Ass} I^{n^{*}} / I^{n}$.

ProOF. (4.15.1) By (2.2.7), let $k$ be large enough that $\left(I^{n+k}: I^{k}\right)=I^{n^{*}}$. An easy exercise using (2.2.1) shows that $\left(I^{m^{*}}\right)\left(I^{n-m^{*}}\right) \subseteq I^{n^{*}}$. Thus

$I^{n-m^{*}} \subseteq\left(I^{n^{*}}: I^{m^{*}}\right) \subseteq\left(I^{n^{*}}: I^{m}\right)=\left(\left(I^{n+k}: I^{k}\right): I^{m}\right)=\left(I^{n+k}: I^{m+k}\right) \subseteq I^{n-m^{*}}$,

by (2.2.7). Equality holds throughout, proving (4.15.1). 
(4.15.2) If $n=1$, there is no problem. Let $n>1$. If $P \in \operatorname{Ass} R / J$, write $P=(J: c)$ with $c \in R-J$. As $c \in(J: P) \subseteq(J: I) \subseteq\left(I^{n^{*}}: I\right)=I^{n-1^{*}}$ by (4.15.1), we have $P \in \operatorname{Ass} I^{n-1^{*}} / J$, as desired.

(4.15.3) Let $P \in \operatorname{Ass} R / I^{n^{*}}$. We want $P \in \operatorname{Ass} R / I^{n+1^{*}}$. It does no harm to assume that $R$ is local at $P$. Write $P=\left(I^{n^{*}}: c\right)$. Thus $P \subseteq\left(\left(I^{n^{*}}\right) I^{*}: c I^{*}\right) \subseteq$ $\left(I^{n+1^{*}}: c I^{*}\right)$. We claim that $\left(I^{n+1^{*}}: c I^{*}\right)$ is a proper ideal, and hence contained in $P$. This claim will then show that $P=\left(I^{n+1^{*}}: c I^{*}\right)$, so that $P \in \operatorname{Ass} R / I^{n+1^{*}}$, as desired. To prove the claim, suppose it is false. Then $c \in\left(I^{n+1^{*}}: I^{*}\right)=I^{n^{*}}$ by (4.15.1). This contradicts that $c \in R-I^{n^{*}}$.

(4.15.4) Suppose $P \in S_{n}(I)$. We need $P \in$ Ass $I^{n^{*}} / I^{n}$. We may assume that $R$ is local at $P$. Write $P=\left(I^{n}: c\right)$. If $c \notin I^{n^{*}}$, then $\left(I^{n^{*}}: c\right)$ is proper, and hence contained in $P$. Thus $P=\left(I^{n^{*}}: c\right)$ so $P \in \operatorname{Ass} R / I^{n^{*}}$. By (4.15.3) and (2.2.5), $P \in A^{*}(I)$, contradicting that $P \in S_{n}(I)$. Therefore $c \in I^{n^{*}}$, so that $P \in \operatorname{Ass} I^{n^{*}} / I^{n}$.

(4.16) REMARK. Combining (2.2.5) with (4.15.2) easily shows that for all sufficiently large $n$, Ass $R / I^{n}=\operatorname{Ass} I^{n-1} / I^{n}$. (This is not necessarily true if $I$ is not regular.)

\section{Examples.}

(5.1) EXAMPLE. Let $R=F\left[X, Y, Z_{1}, \ldots, Z_{n}\right]$ with $F$ a field and $X, Y, Z_{1}, \ldots$, $Z_{n}$ indeterminates, and with $n \geq 1$. Let $Q=(X, Y) R$, and let $W$ be a finite set of primes of $R$ with each $P \in W$ properly containing $Q$. Then there is an ideal $K$ projectively equivalent to $Q$ such that Ass $R / K^{2}=$ Ass $R / K^{3}=\cdots=\{Q\}$, while Ass $R / K=W \cup\{Q\}$ (so that $S(K)=S_{1}(K)=W$ ). (Note that in this example, $Q^{n}=Q^{n^{*}}$ for all $n \geq 1$.)

PROOF. Let $T=F[X, Y]$, and let $q=(X, Y) T$, so that $R=T\left[Z_{1}, \ldots, Z_{n}\right]$ and $Q=q R$. Let $H=\left(X^{3}, Y^{3}\right)\left(X^{3}, Y^{3}, X^{2} Y\right) T$, an ideal in $T$. By (3.3) applied to $(X, Y) T, H_{q} \neq H_{q}^{*}$, and $H$ is projectively equivalent to $q=(X, Y) T$. Using (2.2.5), we can take $m$ to be the largest integer for which $H_{q}^{m} \neq H_{q}^{m^{*}}$. Let $I=H^{m} R$. We claim that $I$ only has one prime divisor, namely $Q$. As is well known, $Q^{\prime}$ is a prime divisor of $I=H^{m} R=H^{m} T\left[Z_{1}, \ldots, Z_{n}\right]$, if and only if $Q^{\prime}=q^{\prime} R$ for some prime $q^{\prime}$ of $T$ with $q^{\prime}$ a prime divisor of $H^{m}$. However, $q$ is the only prime of $T$ which contains $H$, hence the only prime divisor of $H^{m}$, and so our claim follows. We next claim that $I_{P} \neq I_{P}^{*}$ for all $P \in W$. Since $R$ is a flat extension of $T$, the definition of $H^{m^{*}}$ shows that $I^{*}=\left(H^{m} R\right)^{*}=H^{m^{*}} R$. Since $H^{m} \neq H^{m^{*}}$ (since this holds upon localizing at $q), I^{*} \neq I$. Now for an ideal $J$, it is easily seen that $J \neq J^{*}$ if and only if $J_{P} \neq J_{P}^{*}$ for some prime divisor $P$ of $J$. As $Q$ is the only prime divisor of $I$, we must have $I_{Q} \neq I_{Q}^{*}$. For any $P \in W$, we have $Q \subset P$, and so it follows that $I_{P} \neq I_{P}^{*}$, proving our second claim. This says that $\left(I: I^{*}\right) \subseteq P$ for all $P \in W$. Thus we have shown that $I$ and $W$ satisfy one of the hypotheses of (2.7). Since $\left(I: I^{*}\right)=\left(H^{m} R:\left(H^{m} R\right)^{*}\right)=\left(H^{m} R: H^{m^{*}} R\right)=\left(H^{m}: H^{m^{*}}\right) R$, clearly $Q=q R$ is the only prime of $R$ minimal over $\left(I: I^{*}\right)$. As every $P \in W$ properly contains $Q$, a second hypothesis of (2.7) is satisfied. Furthermore, obviously $A^{*}\left(H^{m}\right)=\{q\}$, and so $A^{*}(I)=\{Q\}$. Therefore $W \cap A^{*}(I)=\varnothing$, satisfying the third hypothesis of (2.7). By that result, there is an ideal $K$ with $I \subseteq K \subseteq I^{*}$, and with $S_{1}(K)=W$.

We now show that Ass $R / K^{2}=$ Ass $R / K^{3}=\cdots=\{Q\}$. Let $k \geq 2$. From $H^{m} R=I \subseteq K \subseteq I^{*}=H^{m^{*}} R$, we see that $H^{m k} R=I^{k} \subseteq K^{k} \subseteq\left(I^{*}\right)^{k}=$ 
$\left(H^{m^{*}}\right)^{k} R \subseteq\left(H^{m k}\right)^{*} R$. However, by the choice of $m, H_{q}^{m k}=H_{q}^{m k^{*}}$, and since $q$ is the only prime divisor of $H^{m k}$, we must have $H^{m k}=H^{m k^{*}}$. Combining this with the above shows that $K^{k}=I^{k}=H^{m k} R$ for all $k \geq 2$. Since $Q$ is the only prime divisor of $H^{m k} R$, we have Ass $R / K^{k}=\{Q\}$ for all $k \geq 2$, as desired. Since $S_{1}(K)=W$, and since $Q \in \operatorname{Ass} R / K(Q$ being minimal over $K)$, we see that Ass $R / K=W \cup\{Q\}$. It only remains to show that $K$ is projectively equivalent to $Q$, which follows from $H$ being projectively equivalent to $q$.

(5.2) ExAmple. Let $F$ be a field and $X$ be an indeterminate. Let $R=$ $F+X^{3} F[X]$. Let $P=X^{3} F[X]$, and let $I=\left(X^{3}, X^{4}\right) R$. Then $I \neq I^{*}=(I)_{a}=P$, while for all $n \geq 2, I^{n}=I^{n^{*}}=\left(I^{n}\right)_{a}=P^{n}$. Also, in $\mathbf{R}=\mathbf{R}(I),(u, P, I t) \mathbf{R}$ is an irrelevant prime divisor of $u \mathbf{R}$. Furthermore, $u \mathbf{R}$ also has a relevant prime divisor lying over $P$.

PrOOF. Note that $P=\left(X^{3}, X^{4}, X^{5}\right) R$. Also, since $X^{3} R$ is principal, its integral closure is $X^{3} F[X] \cap R=P$, using that $F[X]$ is the integral closure of $R$. Since $X^{3} R \subseteq I \subseteq P$, we see that $(I)_{a}=P$. Now $I^{2}=\left(X^{6}, X^{7}, X^{8}\right) R=P^{2}$, so that by (2.2.1) and (2.2.4), $P=I^{*}$. Clearly $I \neq I^{*}$, since $X^{5} \notin I$. Now let $n \geq 2$. Then $I^{n}=\left(X^{3 n}, X^{3 n+1}, X^{3 n+2}\right) R=P^{n}$. This also equals $X^{3 n} F[X] \cap R=\left(X^{3 n} R\right)_{a}$. As $X^{3 n} R \subseteq I^{n}=\left(X^{3 n} R\right)_{a}$ we have $\left(I^{n}\right)_{a}=I^{n}$. By (2.2.4), $I^{n^{*}}=I^{n}$.

Since $P^{2}=I^{2} \subseteq I$, we have $P \subseteq(I: P)$. Now $P$ is maximal, and $(I: P)$ is proper. Thus $P=(I: P)=\left(I: I^{*}\right)$. By $(4.12),(u, P, I t) \mathbf{R}$ is a prime divisor of $u \mathbf{R}$. Furthermore, since height $P=1$, clearly $P \in A^{*}(I)$, and so (4.3.2) shows that $u \mathbf{R}$ also has a relevant prime divisor lying over $P$.

(5.3) REMARK. An attempt at a converse to (4.10) would read, if for some $n \geq 1$ and all $k \geq 0, P \in$ Ass $I^{n+k-1} / I^{n+k}$, then there is a $b \in R$ with $P=\left(I^{n}: b\right)$ and with $(u, P, I t) \mathbf{R}$ not minimal over $u^{n} \mathbf{R}: b R$. We show that this is false.

Let the notation be as in (5.2). Now $P=\left(I: X^{5}\right)$, so $P \in$ Ass $R / I$. Also, for $k \geq 2, P=\left(I^{k}: X^{3(k-1)}\right)$, so $P \in \operatorname{Ass} I^{k-1} / I^{k}$. Therefore, with $n=1$, we have $P \in$ Ass $I^{n+k-1} / I^{n+k}$ for all $k \geq 0$. To produce our counterexample, we must show that for any $b \in R$, if $P=(I: b)$, then $(u, P, I t) \mathbf{R}$ is minimal over $u \mathbf{R}: b \mathbf{R}$. We will in fact show that these two ideals are equal. Let $m<0$. Then the component of degree $m$ of $u \mathbf{R}: b \mathbf{R}$ is just $R t^{m}$, which is also the component of degree $m$ of $(u, P, I t) \mathbf{R}$. If $m=0$, the component of $u \mathbf{R}: b \mathbf{R}$ of degree 0 is $(I: b)$, while the component of $(u, P, I t) \mathbf{R}$ of degree 0 is $P$. By choice of $b$, these are equal. For $m>0$, the component of $u \mathbf{R}: b \mathbf{R}$ of degree $m$ is $\left(\left(I^{m+1}: b\right) \cap I^{m}\right) t^{m}$, while for $(u, P, I t) \mathbf{R}$, that component is $I^{m} t^{m}$. We must show that these are equal. Thus, we need $I^{m} \subseteq\left(I^{m+1}: b\right)$. We first claim that $b \in P$. Since $P=(I: b), b X^{5} \in I$. This shows that $b$ has no constant term, which proves the claim. Now $I^{m} b \subseteq I^{m} P$. However, we have $I^{m+1} \subseteq I^{m} P \subseteq P^{m+1}=I^{m+1}$, the equality from (5.2). Thus $I^{m} P=I^{m+1}$, and so $I^{m} b \subseteq I^{m+1}$, showing $I^{m} \subseteq\left(I^{m+1}: b\right)$ as desired.

(5.4) REMARK. In (5.2), we have $P=\left(I: X^{5}\right)$, and $X^{5} \in P=I^{*}$ so that $P \in \operatorname{Ass} I^{*} / I$. However, $P \in A^{*}(I)$, so $P \notin S_{1}(I)$. Thus, in (4.15.4), the inclusion may be proper.

(5.5) EXAMPLE. Let $F$ be a field and let $X$ and $Y$ be indeterminates. Let $R=F+\left(X^{3}, Y\right) F[X, Y]$. Let $P=\left(X^{3}, Y\right) F[X, Y] \cap R$, and let $I=\left(X^{3}, X^{4}\right) R$. Then $I \neq I^{*}=(I)_{a}$, but for all $n \geq 2, I^{n}=I^{n^{*}}=\left(I^{n}\right)_{a}$. Also, $P \notin$ Ass $R / I^{n}$ except when $n=1$. Furthermore, in $\mathbf{R}=\mathbf{R}(I),(u, P, I t) \mathbf{R}$ is an irrelevant prime 
divisor of $u \mathbf{R}$, and is the only prime divisor of $u \mathbf{R}$ lying over $P$. (This example is taken from [B].)

PrOOF. Since $X^{3} R \subseteq I \subseteq\left(X^{3}, X^{4}, X^{5}\right) R=\left(X^{3} R\right)_{a}$, we have

$$
(I)_{a}=\left(X^{3}, X^{4}, X^{5}\right) R \neq I \text {. }
$$

However, $I^{2}=\left(X^{6}, X^{7}, X^{8}\right) R=\left((I)_{a}\right)^{2}$. By $(2.2 .1)$ and $(2.2 .4), I^{*}=(I)_{a}$. For $n \geq 2$, we easily see that $I^{n}=\left(X^{3 n}, X^{3 n+1}, X^{3 n+2}\right) R=\left(I^{n}\right)_{a}=\left(X^{3 n} R\right)_{a}$. By (2.2.4), $I^{n}=I^{n^{*}}=\left(I^{n}\right)_{a}$. Now it is easily seen that $P=\left(I: X^{5}\right)$ but $P \notin$ Ass $R / I^{n}$ for $n \geq 2$. By (4.4.3), $(u, P, I t) \mathbf{R}$ is the only prime divisor of $u \mathbf{R}$ lying over $P$.

(5.6) REMARK. Let $P \in$ Ass $R / I^{n}$ but $P \notin$ Ass $R / I^{n+1}$. Then (4.11.1) shows that $(u, P, I t) \mathbf{R}$ is a prime divisor of $u \mathbf{R}$. The easiest examples of this have $P \in$ $S(I)$, as in (5.5). It is harder to give an example with $P \notin S(I)$. For that, we need $P \in$ Ass $R / I^{n}$ and $P \in A^{*}(I)$, but $P \notin$ Ass $R / I^{n+1}$. R. Cowsik has an (unpublished) example of this behavior. (If we drop the restriction that $I$ is regular, examples are easier.)

\section{REFERENCES}

[B] M. Brodmann, Asymptotic stability of Ass $\left(R / I^{n}\right)$, Proc. Amer. Math. Soc. 74 (1979), 37-39.

[HO] M. Herrmann and U. Orbanz, On equimultiplicity, Math. Proc. Cambridge Philos. Soc. 91 (1982), 207-213.

[KR] D. Katz and L. J. Ratliff, Jr., U-essential prime divisors and sequences over an ideal, Nagoya J. Math. 103 (1986), 39-66.

[M] S. McAdam, Asymptotic prime divisors, Lecture Notes in Math., vol. 1023, Springer-Verlag, Berlin and New York, 1983.

[MR] A. Mirbagheri and L. J. Ratliff, Jr., On the relevant transform and the relevant component of an ideal, 27 page preprint.

[N] M. Nagata, Local rings, Interscience Tracts 13, Interscience, New York, 1962.

[NR1] D. G. Northcott and D. Rees, Reductions of ideals in local rings, Math. Proc. Cambridge Philos. Soc. 50 (1954), 145-158.

[NR2] _,$A$ note on reductions of ideals with an application to the generalized Hilbert functions, Math. Proc. Cambridge Philos. Soc. 50 (1954), 353-359.

[R] L. J. Ratliff, Jr., On prime divisors of $I^{n}, n$ large, Michigan Math. J. 23 (1976), 337-352.

[RR] L. J. Ratliff, Jr. and D. E. Rush, Two notes on reductions of ideals, Indiana Univ. Math. J. 17 (1978), 929-934.

[S] V. M. Smith, Strongly superficial elements, Pacific J. Math. 58 (1975), 643-650.

Department of Mathematics, University of Texas, Austin, Texas 78712

Department of Mathematics, University of California, Riverside, CaliforNIA 92521 